\title{
Parameter Optimization Technique Using The Response Surface Methodology
}

\author{
K. Koyamada ${ }^{1}$, K. Sakai ${ }^{1}$, T. Itoh $^{2}$ \\ ${ }^{1}$ Department of Engineering, Kyoto University, Kyoto, Japan \\ ${ }^{2}$ Department of Infomatics, Kyoto University, Kyoto, Japan
}

\begin{abstract}
In this paper, we propose a parameter optimization technique using the Response Surface Methodology (RSM) for the accurate biological cell simulation which calculates cell behavior by numerically integrating the differential equation and generates action potentials, intracellular Ca transient, contraction, and intracellular ATP consumption by using parameter values of major ion permeability and amplitude of Ko dependency. Since most of these parameters cannot be directly measured by experiment, these are searched for by applying an optimization technique, that is, by minimizing an objective function defined as a difference between a measured and a calculated waveform of action potentials in a cardiac myocyte. We employ the RSM as the optimization technique. In the RSM, a quadratic polynomial is used in general, and a mathematical technique is used to calculate the extreme points. Because our parameter space cannot be approximated by a single quadratic polynomial surface, we adopt a recursive subdivision technique and use the coefficient of multiple determination to representing a response surface in each subspace for a criteria of the subdivision. We confirmed the effectiveness of the proposed technique by searching for parameters which are determined in advance.
\end{abstract}

Keywords - Parameter optimization, response surface methodology, biological cell simulation

\section{INTRODUCTION}

Recently, there was development in biological fields, such as biochemistry and molecular biology. So it became possible to analyze life activity functionally from microscopic viewpoints, such as in a gene or a cell, which means that the approach of biology changed fundamentally. With this, a new scientific discipline called Bioinformatics was born. This means analyzing DNA or protein sequences using the methodology of information science. Now, the genetic code of various living things is analyzed using this technique, and the function is becoming clear.

All living bodies are composed of a set of cells. That is, it can be said that a life phenomenon is a collection of reactions occurring in a cell. Development in Bioinformatics field enabled it to analyze the mechanism in a cell in detail. Thereby, research results on life information are accumulated. However, even if each gene and the function of each gene and each substance are known, we cannot necessarily understand behavior of the whole cell. It is because the structure of a cell is formed as the network in which the actions of a gene or a metabolism substance become entangled intricately. In order to understand the structure of the network, a model is assembled based on the data of the collected life information, and it reappears using a computer, that is, reconstruction of life information is needed. This is a cell simulation.

The Heart Simulation Project at Kyoto University aims at unifying the analytic research on a genome, proteome, etc., and searching for a new principle, making full use of the calculation power of a computer system. The project develops a cardiac myocyte model (KYOTO Model [8]), which is composed of the majority of known sarcolemmal ion channels and transporters, sarcoplasmic reticulum, and contractile elements. This model is based on the HodgkinHuxley model, and simulates cell behavior by numerically integrating the differential equation and generates action potentials, intracellular $\mathrm{Ca}$ transient, contraction, and intracellular ATP consumption by using parameter values of major ion permeability and amplitude of Ko dependency. Since most of these parameters cannot be directly measured by experiment, these are identified by experimental results. Parameter identifications of the cardiac myocyte model were regarded as an optimization problem that minimizes an objective function defined as a difference between a measured and a calculated waveform of action potentials in a cardiac myocyte.

AR Willms and et. al. estimated parameters of the Hodgkin-Huxley model by fitting each conductance trace from a voltage-clamp experiment[3]. They employed a standard nonlinear least-squares optimization algorithm to minimize the objective function. Saitoh and et. al. utilized the E-Cell simulation environment as a framework of estimating parameters for the accurate cell simulation by employing the Genetic $\operatorname{Algorithm}(\mathrm{GA})$ in addition to standard optimization algorithms, such as the Rosenbrock method and the Modified Powell method[4]. In the standard optimization algorithm, the quality of the solution is deeply dependent on the selection of the initial values of the parameters to be estimated. Although the GA might solve the problem caused by the initial values, it might take a lot of computational time and could not consider the local distribution of the objective function. To cope with these problems, we employ the Response Surface Methodology (RSM) [1] as the optimization technique. In general, the RSM is used for optimization of the process of a product design etc, and thermal simulation[5,6,7].

In the RSM, a quadratic polynomial is used in general, and a mathematical technique is used to calculate the extreme points. After we evaluate our parameter space, which is composed of three parameters, we understand that no quadratic polynomial surface can approximate our 
parameter space. To solve this problem caused by many local minima, we adopt a recursive subdivision technique and use the coefficient of multiple determination to representing a response surface in each subspace for a criteria of the subdivision. In this paper, first, we describe our parameter optimization technique, and then verify the effectiveness of the proposed technique by searching for parameters which are determined in advance.

\section{METHODOLOGY}

The Kyoto Model is implemented as a computer program, which carries out the simulation of the electric action potential of a cardiac myocyte by using parameter values of major ion permeability and amplitude of Ko dependency. The waveform of the action potential, which we take into consideration during the parameter optimization in this research, is shown in Fig.1. In this research, we optimize three parameters: "Na permeability", "Ca permeability", and "amplitude of Ko dependency."

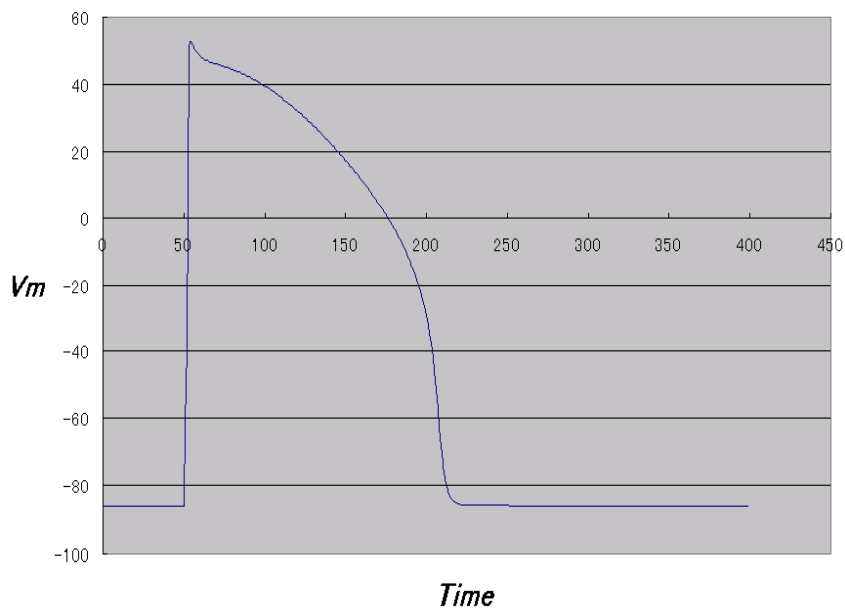

Fig. 1. The waveform of action potential in a cardiac myocyte

We identify these parameters of the Kyoto Model as an optimization problem, which minimizes the difference between a measured and a calculated waveform of action potentials in a cardiac myocyte. In order to check the validity of the proposed technique, we, in advance, determine parameters which generates a waveform used as a target waveform in the Kyoto Model. To search for these parameters (target parameters), we employ the RSM, which is a technique to approximate the unknown response function by using some design points. Here, a design point is a set of parameters. Generally, in the RSM, a secondorder model is used, since it is flexible, and it is easy to estimate the parameters. In general the second-order model is as follows:

$y=\beta_{0}+\sum_{i=1}^{k} \beta_{i} x_{i}+\sum_{i=1, j \leq i}^{k} \beta_{i j} x_{i} x_{j}$ where $\mathrm{k}$ denotes the number of parameters at each design point and $\mathrm{y}, \mathrm{x}_{\mathrm{i}}(i=1, \ldots, k)$ and $\beta_{i}(i=1, \ldots, k)$ represent the known response value, the known parameters and the unknown coefficients, respectively.

In the RSM-based technique, we define a response as an association measure calculated by using a difference between the target waveform and a waveform simulated using a set of parameters. These parameters are determined using the Design of Experiments (DOE)[2]. In this paper, we define an association measure $(\chi)$ as

$\chi=\sum_{t=1}^{400} \sqrt{\left(V_{0}(t)-V(t)\right)^{2}}$

where $V_{0}(t)$ and $V(t)$ represent the target potential at $t(\mathrm{msec})$, and the calculated potential at $t(\mathrm{msec})$, respectively. In short, when the value of $\chi$ equals to zero, the calculated waveform agrees with the target waveform completely. Note that " $\chi$ " is identical to the dependent variable " $y$ " in Eq.(3).

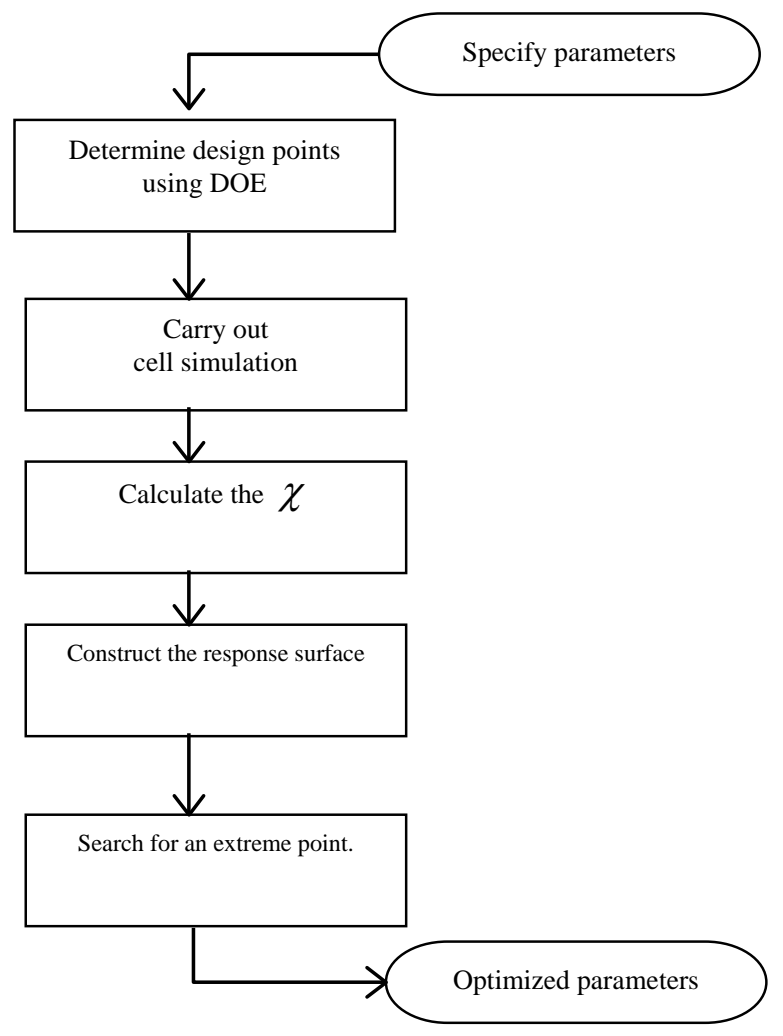

Fig. 2. Identification process using the RSM-based technique

In general, the process of the RSM-based optimization technique is shown in Fig.2. We first specify parameters and the range of their values, and determine some sets of parameters by using the DOE. Secondly, we carry out the cell simulation using sets of parameters determined by DOE, and calculate the value of $\chi$ by using the calculated and target waveforms. Note that the number of times that the cell 
simulation are carried out is the same as that of design points. Finally, we construct the response surface and search for an extreme point.

By evaluating our parameter space in the full combinatorial design of three parameters, we understand that a single response surface cannot approximate our parameter space. It means that a simple polynomial is not recommended with respect to the accuracy of parameter optimization results. It must be noted that there may be multiple parameter sets that generate a waveform equally well fitted to the target waveform.

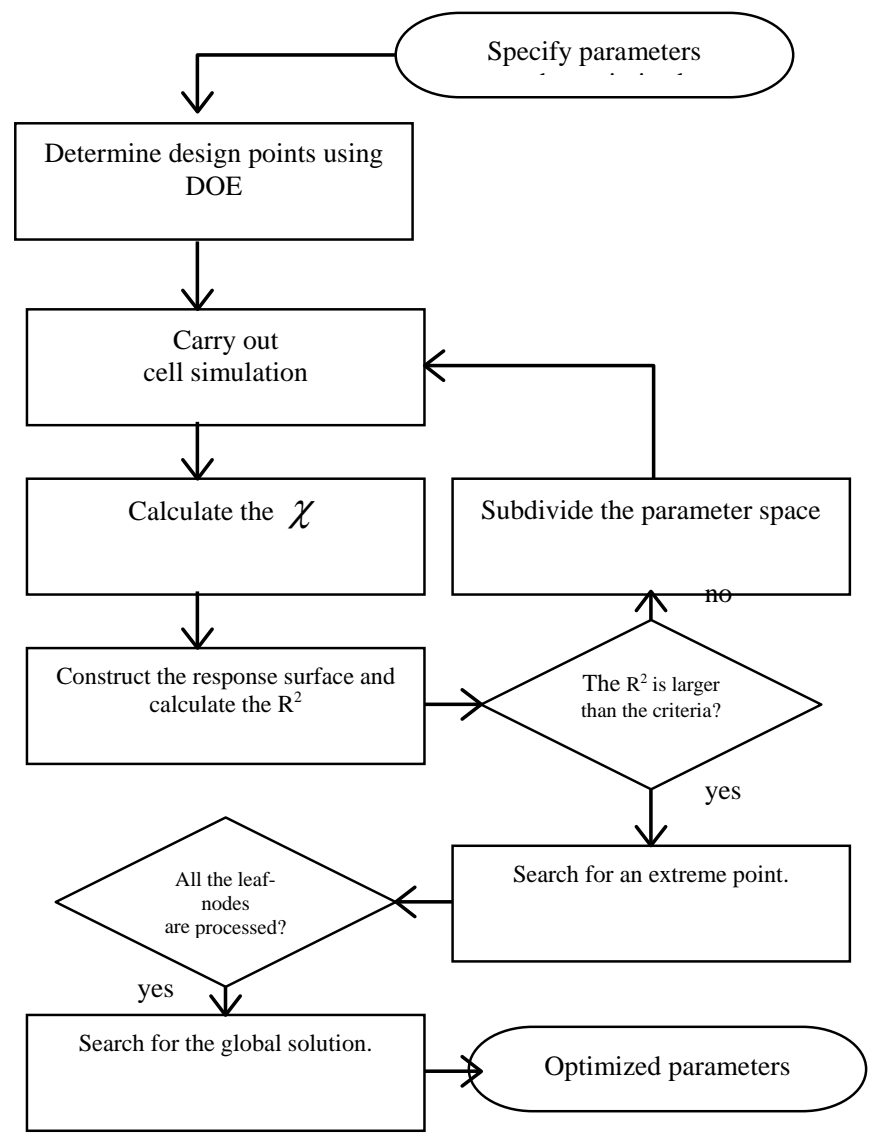

Fig. 3. Optimization process by recursive subdivision technique

In general, the distribution of $\chi$ is too complex to be presented by a single response surface in the parameter space. To solve this problem, we propose an RSM-based optimization technique using a recursive subdivision strategy. To determine whether further subdivision should be made, we employ an coefficient of multiple determination $\left(\mathrm{R}^{2}\right)$ which shows the degree of adaptation of a response surface. The $R^{2}$ is defined as,

$$
\mathrm{R}^{2}=1-\frac{S S_{R} /(k-p-1)}{S_{y y} /(k-1)}
$$

where $k$ and $p$ denotes the number of parameters and responses, and $S S_{R}$ and $S_{Y Y}$ represent regression sum of squares, and total sum of squares, respectively. If each parameter is normalized, this value will be between 0 and 1.The high or low value means that the response surface created by RSM is well adapted or not, respectively.

With the physiological viewpoint of the, the range of each parameter was determined as follows.

- "Na permeability" $\quad 2000 \sim 3000$

- "Ca permeability" $7000 \sim 9000$

- "Amplitude of Ko dependency" $100 \sim 200$

The process of the proposed optimization technique is shown in Fig.3. In our proposed optimization technique, we first construct a response surface in the whole parameter space, and calculate the $\mathrm{R}^{2}$ of the response surface. If the calculated coefficient is under the user-specified criteria, we subdivided the space by splitting each parameter range into two at equal intervals. For example, in the case of three parameters like our experimental case, the parameter space will be subdivided into $2^{3}=8$ subspaces. Each subspace is recursively subdivided until the calculated coefficient of the subspace is beyond the criteria. We call a subspace with the satisfied coefficient as a leaf-subspace, that is a leaf-node of the parameter tree. In the leaf-subspace, the response surface is well adopted, and we search for the local optimum solution, that is a set of parameters which minimize the value of $\chi$. When the $\mathrm{R}^{2}$ of the response surface exceeds the criteria in each of the leaf-subspaces, we search for a global solution among all the local solutions in the leafsubspaces.

\section{RESULTS AND DISCUSSION}

Two metrics may be considered to verify the accuracy of a set of parameters which are searched for by our optimization technique. The first is the distance between the calculated and exact solution, and the second is the value of $\chi$ evaluated by using the calculated solution. Since in general, the exact solution may not be known in advance, the second metric is adopted in this research.

Table. 1 shows the $\mathrm{R}^{2}$ and the optimal solution in each subspace after the first subdivision. Here, we specify the user-specified criteria for the further subdivision as 0.9.In Table.1, the combination of the number of the leftmost column means the location of the relevant subspace. The first, second, or third number represents "Na permeability", "Ca permeability", or "Amplitude of Ko dependency", respectively. For example, "1-2-1" means the following subspace.

- "Na permeability" $\quad 2000 \sim 2500$

- "Ca permeability" $8000 \sim 9000$

- "Amplitude of Ko dependency" $100 \sim 150$

Since the $\mathrm{R}^{2}$ are beyond the user-specified criteria, 0.9 , in the subspaces "1-1-2", "1-2-1", "2-1-2", and "2-2-1", optimal solutions are calculated. On the other hand, since they are below the criteria in the subspaces, "1-1-1", "1-2-2", 
"2-1-1", and "2-2-2", the further subdivisions are made. So in Table.1, the $\mathrm{R}^{2}$ are shown only in those subspaces.

As shown in Fig.3, the recursive subdivision is made until the $\mathrm{R}^{2}$ are beyond the user-specified criteria in all the leafsubspace. Table. 2 shows the optimization result. In this experiment, the recursive subdivision was performed at four stages. This corresponds to the case in which each parameter range is equally split into sixteen.

TABLE I

Result of first recursive subdivision

\begin{tabular}{|c|c|c|c|c|c|}
\hline $\begin{array}{c}\text { subdivision } \\
\text { pattern }\end{array}$ & $\mathrm{Na}$ & $\mathrm{Ca}$ & IK1 & $\begin{array}{c}\text { association } \\
\text { measure }\end{array}$ & $\begin{array}{c}\text { coefficient of } \\
\text { multiple } \\
\text { determinations }\end{array}$ \\
\hline $1-1-1$ & & & & & 0.725354 \\
\hline $1-1-2$ & 2000 & 7800.939 & 150 & 36.06178 & $0.9<$ \\
\hline $1-2-1$ & 2244.676 & 9000 & 150 & 58.21841 & $0.9<$ \\
\hline $1-2-2$ & & & & & 0.728439 \\
\hline $2-1-1$ & & & & & 0.8004 \\
\hline $2-1-2$ & 2500 & 7809.199 & 150 & 24.35524 & $0.9<$ \\
\hline $2-2-1$ & 2744.897 & 9000 & 150 & 69.61225 & $0.9<$ \\
\hline $2-2-2$ & & & & & 0.6745 \\
\hline
\end{tabular}

TABLE II

Comparison of optimization results

\begin{tabular}{|c|c|c|c|c|}
\hline & $\mathrm{Na}$ & $\mathrm{Ca}$ & $\mathrm{IK} 1$ & $\begin{array}{c}\text { association } \\
\text { measure }\end{array}$ \\
\hline All range(quadratic) & 2000 & 8869.301 & 149.8869 & 44.71859 \\
\hline All range(quartic) & 2000 & 7902.233 & 149.0073 & 24.87055 \\
\hline Recursive subdivision & 2312.5 & 7625 & 140.494 & 0.488175 \\
\hline Exact solution & 2300 & 7600 & 140 & 0 \\
\hline
\end{tabular}

For comparison, we optimize parameters without the recursive subdivision, that is using a single response surface. In Table 2, the first and the second rows show the optimized parameters and the relevant values of $\chi$ using the quadratic and quartic polynomial-based response surfaces,

respectively. The proposed technique, listed in the third row, is quadratic polynomial-based, and superior to these results with respect to the value of $\chi$.

In general, if we assume that

1. $\quad k$ parameters are optimized

2. $\quad \boldsymbol{M}$ stages are required to obtain optimized parameters

by using our technique, and

3. In each stage, the $\mathrm{R}^{2}$ become beyond the user-specified criteria ( 0.9 in this experiment) in the half of the whole subspaces at the stage,

the improvement ratio will be

$$
\frac{\left(2^{k-1}-1\right) \times 2^{k \times(M-1)}}{2^{M \times(k-1)}-1}
$$

\section{CONCLUSION}

In this paper, we propose an RSM-based optimization technique using a recursive subdivision. The point is to use an $\mathrm{R}^{2}$ which shows the degree of adaptation of a response surface in order to determine whether to proceed further subdivision or not. We apply this technique for optimizing three parameters for improving the accuracy of biological cell simulation and confirm its effectiveness. In addition, we show a theoretical equation for evaluating the performance improvement of our technique.

\section{REFERENCES}

[1]. Raymond H. Myers, Douglas C. Montgometry, "Response Surface Methodology", John Wiley \& Sons. Inc, 1995

[2]. Douglas C. Montgomery, "Design and Analysis of Experiments 4th edition", John Wiley \& Sons Inc, 1996

[3]. AR Willms, DJ Baro, RM Harris-Warrick, and J. Guckenheimer. "An Improved Parameter Estimation Method for Hodgkin-Huxley Models" J. Comp. Neurosci. vol.6, pp145-168, 1999.

[4]. Saito,Y.,Takahashi,K.,Iwata,T.,Aikawa,T. and Tomita,M.,Parameter estimation mechanism of E-CELL simulation environment, Genome Informatics, 10:358-359,1999.

[5]. Kuzuno, M, Nishio, T., and Koyamada, K., and Nishio, T.,"A thermal compact modeling method using Response Surface Methodology with Genetic Algorithm," PDPTA, CISST \& IC-AI International Conferences, pp. 627-634, 2000

[6]. Kuzuno, M, Nishio, T., and Koyamada, K.,"Compact modeling for Thermal Simulation Using Response Surface Methodology," International Symposium on Computational Technologies for Fluid/Thermal/Chemical Systems With Industrial Applications," PVP-Vol. 424-2, pp. 57-63, 2001

[7]. Okamoto, K., Koyamada, K., Kuzuno, M., and Nishio, T., "A technique for developing a precise thermal compact model," 4th International Symposium on Computational Technologies for Fluid/Thermal/Chemical Systems with Industrial Applications, PVPVol. 448-2, pp. 259-265, 2002

[8]. Satoshi MATSUOKA, Nobuaki SARAI, Shinobu KURATOMI, Kyoichi ONO, and Akinori NOMA, "Role of Individual Ionic Current Systems in Ventricular Cells Hypothesized by a Model Study", Japanese Journal of Physiology, 53, PP.105-123, 2003 